\title{
Use of IR Thermography for Bioheat Transfer Studies
}

Boris Vainer ${ }^{\text {a) }}$, Petr Belozerov ${ }^{\text {a) }}$ and Victor Baranov ${ }^{\text {b) }}$

a) Institute of Semiconductor Physics, Russian Academy of Sciences, Siberian Branch,

13, Lavrentyev avenue, Novosibirsk, 630090, Russia

Tel.: +(7) 3832333506 , Fax: +(7) 3832332926 , E-mail: bgv@isp.nsc.ru

b) State-Research Institute of Physiology, Russian Academy of Medical Sciences, Siberian Branch

Keywords: Bioheat transfer, Blood perfusion, Pathologies, Computer simulation, Infrared thermography.

Preference: Oral presentation.

Around the world, extensive studies on bioheat transfer are under way; bioheat transfer mechanisms both in deep and in superficial tissues are of interest. Over years of research, various models for the process have been proposed. Presently available bioheat-transfer models enable adequate reconstruction of 3D temperature fields inside bodies at depths up to $3 \mathrm{~cm}$ from measured skin-temperature distributions, providing means quite sufficient for solving many practical medical and biological problems. Normally, input data for modelling are provided by skin temperature measurements. To gain maps of skin temperature, various contact techniques have found acceptance, based on such instruments as thermocouples, thermistors, liquid-crystal film indicators, etc. In some cases, temperatures are sampled directly from deep tissues; yet, this strategy suffers many artefacts resulting from the invasiveness of the procedure and its incapability to preserve the natural functioning of the tissue. In the abovementioned investigations, IR thermography technique may offer many advantages in solving the problems since it permits contactless panoramic measurements, simultaneously minimizing the outside intervention into organism's functioning. 
The purpose of the present study was to develop an IR thermography method for contactless probing of the 3D temperature field inside bodies, considering the fact that a computerized IR-imager allows fast examination of the skin temperature fields on the surface of living bodies and consequently easy identification of heat-exchange mechanisms in their tissues. This offers broad potentialities in medical diagnostics, including cases where data are to be taken from arbitrarily chosen persons (in screening tests, for instance).

First bioheat transfer models date backs to the 1940s. In 1948 , the pioneering article by H.H.Pennes appeared in Journal of Applied Physiology [1]. In this paper, H.H.Pennes proposed a model to describe the effects of metabolism and blood perfusion on the energy balance within tissue, based on the socalled "bioheat transfer equation"

$$
\rho c \frac{d T}{d t}=k \nabla^{2} T+\rho_{b} c_{b} \omega_{b}\left(T_{a}-T\right)+q_{m e t}
$$

where $\rho, c$, and $k$ are respectively the density, the specific heat, and the thermal conductivity of the tissue; $\rho_{b}$ and $c_{b}$ denote density and specific heat of blood; $\omega_{b}$ is the blood perfusion rate in the tissue; $q_{m e t}$ is the metabolic heat generation; $T_{a}$ is the arterial blood temperature; and $T$ is the tissue temperature. Over many years, this model has been many times improved and refined for particular cases, put under considerable criticism, and developed (M.Chen, K.Holmes; S.Wienbaum, L.Jiji, D.Lemons; G.Anderson, J.Valvano; E.Wissler et al.)

The calculations made in the present study were mainly based on the Pennes equation due to its simplicity, on the one hand, and normally sufficiently high adequacy, on the other. This equation permits fast calculations of $3 D$ temperature fields from skin-temperature maps, this merit going well with the capability of the IR-imaging technique to ensure high data acquisition speed. In the approximation provided, adequate preliminary analysis of subcutaneous temperature distributions is possible, followed by subsequent more detailed examination of 3D anomalies, if any. In the latter case, the modified form of the equation was devised. 
The temperature maps obtained with the help of an IR imager were processed on a computer by solving the bioheat transfer equation. A good agreement between theoretically predicted and experimental data for various combinations of parameters was obtained. Numerical and natural experiments with tissues affected by some pathological processes and/or irritated by some external and internal factors were performed.

Figure 1 shows the calculated typical in-depth profiles of temperature in a tissue for situations in which an internal heat source was positioned inside the body at two different depths $X_{S}$, for the case of fixed blood perfusion rate $\omega_{b}=0.0005$ $(\mathrm{ml} / \mathrm{s}) / \mathrm{ml}$. In solving the problem, Newtonian boundary conditions for convective heat transfer in air over body skin were used. As in previously reported studies, the core temperature was assumed constant. In the calculations, the parameters were as follows: $T_{a}=38{ }^{\circ} \mathrm{C}$, ambient temperature $25{ }^{\circ} \mathrm{C}, k=0.5 \mathrm{~W} /\left(\mathrm{m}^{\circ} \mathrm{C}\right), \quad c=c_{b}=4200 \mathrm{~J} /\left(\mathrm{kg}{ }^{\circ} \mathrm{C}\right), q_{m e t}=400$ $\mathrm{W} / \mathrm{m}^{3}$. The graph of Figure 1 is indicative of high rate of bioheat outflow from the superficial layer of the tissue (resulting in a more sloping behaviour of the curve at shallower depths). The temperature gradient decreases with depth, clearly showing that the heat flux from the core also decreases. The notable change in the surface temperature, $\Delta T$, proves the IR-imager to be an adequate tool for analyzing 3D temperature fields inside living organisms.

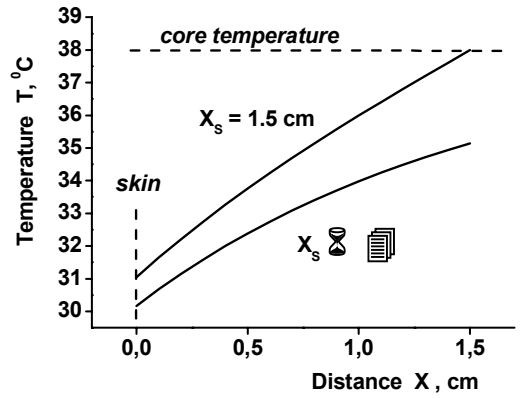

Figure 1.

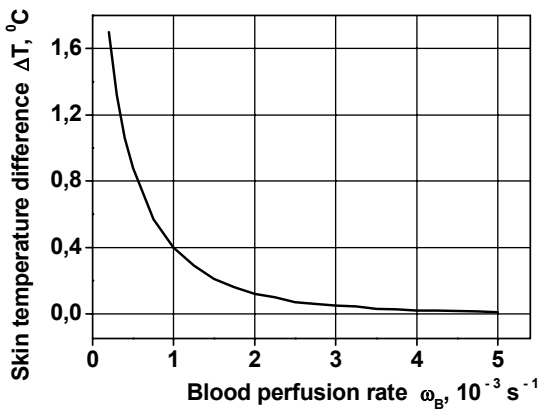

Figure 2. 
Normal and pathological functioning of blood circulation system was modelled in particular by varying the parameter $\omega_{b}$. Blood perfusion in tissues largely affects the distribution of skin temperature over the skin surface. This is evident from Fig. 2 that shows $\Delta T$ versus blood perfusion rate.

\section{References}

1. H.H.Pennes, "Analysis of Tissue and Arterial Blood Temperature in the Resting Human Forearm," J. of Applied Physiology, Vol. 1, 1948, pp. 93-102. 